\title{
Etude des solutions d'équations différentielles du second ordre dépendant d'un paramètre complexe de grand module.
}

\author{
par J. Lombet - GofFAR (à Liége)
}

Résumé. - Du comportement asymptotique des fonctions de Whittaker, on déduit, dans tout l'intervalle fini $[\alpha, \beta]$ décrit par la variable réelle $x$, le comportement de deux solutions linéairement indépendantes des équations différentielles dépendant d'un paramètre complexe $\lambda$ de grand module, dont les «réduites" se ramènent par une transformation de Liouville d̀ l'équation confluente de Whittaker. Le coefficient de la plus haute puissance de $\lambda$ dans l'équation donnèe possède dans $[\alpha, \beta]$, un zéro d'ordre pair $2 \vee$; les cas $\vee=1$ $\checkmark$ pair, $\vee$ impair $(\nu>1)$ sont étudiés séparément.

\section{I. - Position du problème.}

1. - Considérons l'équation différentielle du second ordre

$$
u^{\prime \prime}-\left[a(x) \lambda^{2}+b(x) \lambda+c(x)+X(x, \lambda)\right] u=0 ;
$$

$\lambda$ désigne un paramètre complexe tel que $|\lambda| \geq \Lambda\left(^{1}\right)$ et la variable réelle $x$ décrit l'intervalle $[\alpha, \beta]$ où $\alpha$ et $\beta$ sont deux constantes finies $(\alpha<0<\beta)$.

La fonction $X(x, \lambda)$ est une fonction continue de $x$ dans $[\alpha, \beta]$, développable en une série de puissances de $\lambda^{-1}$ uniformément convergente ponr $|\lambda| \geq \Lambda$ et elle vérifie la condition $X(x, \infty)=0$.

La fonction $a(x)$ continue dans $[\alpha, \beta]$ possède en $x=0$, un zéro d'ordre $2 v, v$ entier positif ou nul $\left({ }^{2}\right)$.

Nous nous proposons d'étudier les équations (1) dont la «réduite»

$$
v^{\prime \prime}-\left[\alpha(x) \lambda^{2}+b(x) \lambda+c(x)\right] v=0
$$

se ramène à

$$
\frac{d^{2} \eta}{d z^{2}}+\frac{1-2 \mu}{z} \frac{d \eta}{d z}+\left(\frac{k}{z}-\frac{1}{4}\right) \eta=0
$$

par une transformation du type

$$
z=f(x, \lambda), \quad v=\eta(z) \psi(x) .
$$

Les fonctions $f(x, \lambda)$ et $\psi(x)$ que nous allons déterminer doivent posséder sur $[\alpha, \beta]$ des dérivées par rapport à $x$ des deux premiers ordres au moins; $k$ désigne une constante éventuellement complexe.

(1) $|\lambda| \geq \Lambda$ signifie que le a module de $\lambda$ est suffisamment grand.

(2) Le fait de supposer que le zéro de $a(x)$ se trouve en $x=0$ et non en $x=x_{0}$ ne nuit en rien à la généralité. 
2. - La substitution (4) transforme l'equation différentielle (2) en une équation

$$
\frac{d^{2} \eta}{d z^{2}}+\frac{1}{z^{\prime}}\left[\frac{z^{\prime \prime}}{z^{\prime}}+\frac{2 \psi^{\prime}}{\psi}\right] \frac{d \eta}{d z}+\frac{1}{z^{\prime 2}}\left[\frac{\psi^{\prime \prime}}{\psi^{\prime}}-a \lambda^{2}-b \lambda-c\right] \eta=0
$$

identique à (3) si

$$
\begin{aligned}
& \frac{z^{\prime \prime}}{z^{\prime}}+\frac{2 \psi^{\prime}}{\psi}=(1-2 \mu) \frac{z^{\prime}}{z} \\
& \frac{\psi^{\prime \prime}}{\psi}-a \lambda^{2}-b \lambda-c=\left(\frac{k}{z}-\frac{1}{4}\right) z^{\prime 2}
\end{aligned}
$$

En intégrant la relation (6) par rapport à $x$, on obtient si la constante d'intégration vaut $(2 \lambda)^{2 \mu}$,

$$
\psi=\frac{z_{2}^{\frac{1}{-\mu}}}{z^{\prime \frac{1}{2}}} \cdot(2 \lambda)^{\mu} .
$$

On détermine le coefficient $c(x)$ de (2) en identifiant à zéro le terme indépendant de $\lambda$ dans (7); nous avons

$$
c=\frac{\psi^{\prime \prime}}{\psi}
$$

La relation (7) est alors identiquement vérifiée si

$$
\begin{aligned}
& z^{2}=4 a \lambda^{2}, \\
& b \lambda+z \frac{z^{\prime 2}}{z}=0 .
\end{aligned}
$$

Pour simplifier les notations, posons

il vient par suite,

$$
a(x)=g^{2}(x)
$$

$$
z^{\prime}=2 g \lambda
$$

$$
z=2 \lambda G \quad \text { avec } G=\int_{0}^{x} g(s) d s
$$

et

$$
b=-\frac{2 k g^{2}}{G} .
$$


Des formules (8) et (10), on déduit la forme de $f(x, \lambda)$ et de $\psi(x)$

$$
\begin{gathered}
f(x, \lambda)=2 \lambda G(x), \\
\psi(x)=G_{2}^{-\frac{I}{-} \mu(x) g-\frac{1}{2}(x)\left({ }^{3}\right) .}
\end{gathered}
$$

Les relations (9) et (11) déterminent respectivement les coefficients $c(x)$ et $b(x)$ en fonction du coefficient arbitraire $a(x)$ de l'equation (2).

3. - Nous supposerons enfin que la fonction $x^{-2 \nu} a(x) \equiv x^{-2 \nu} g^{2}(x)$ possède une dérivée seconde continue et que l'on peut trouver un facteur constant éventuellement complexe $h$ tel que $h x^{-2 y} g^{2}(x)$ soit réelle et positive.

Dans ces conditions, on a

$$
g^{2}(x) \equiv x^{2 v}\left\{a_{0}+a_{1} x+a_{2}(x) x^{2}\right\}
$$

où. les coefficients $a_{0}, a_{1}$ sont constants $\left(a_{0} \neq 0\right)$ et où $a_{2}(x)$ est une fonction continue de $x$ dans $[\alpha, \beta]$.

Pour les valeurs positives de $x, g^{2}(x)$ est réelle et positive. Pour les valeurs négatives de $x$, on a

$$
\arg g^{2}(x)=\arg x^{2 \nu}=2 v \pi \text {. }
$$

Nous prendrons pour $g(x)$, la racine de $g^{2}(x)$ positive pour les valeurs positives de $x$.

La fonction $G(x)$ peut s'écrire

$$
G(x)=x_{2 \mu}^{\frac{1}{\alpha}}\left\{b_{0}+b_{1} x+b_{2}(x) x^{2}\right\}, \mu=\frac{1}{2(\nu+1)}
$$

où $b_{0}, b_{1}$ sont des constantes et où $b_{2}(x)$ est une fonction continue de $x$ dans $[\alpha, \beta]$.

Lorsque $x$ est positif, les fonctions $g(x)$ et $G(x)$ possèdent done l'argument zéro tandis que pour $x$ négatif, elles ont respectivement les arguments $\pi \nu$ et $\pi(\nu+1)$.

La fonction $\psi(x)$ est réelle, positive et bornée sur $[\alpha, \beta]$. Elle est dófinie en tout point de $[\alpha, \beta]$ [en $x=0$, on a $\left.\psi(o)=\lim _{x \rightarrow 0}\left\{Q^{1 / 2-\mu}(x) \cdot g(x)^{-1 / 2}\right\}\right]$ et possède une dérivée seconde continue. On peut donc écrire

$$
\psi(x)=\delta_{0}+\delta_{1} x+\delta_{2}(x) x^{2}, \delta_{0} \neq 0
$$

$\left.{ }^{3}\right)$ On convient qu'une quantite elevée à une certaine puissance $a$ son argument multi. pliê par cette puissance. 
où $\delta_{0}, \delta_{1}$ sont des constantes et où $\delta_{2}(x)$ désigne une fonction continue de $x$ dans $[\alpha, \beta]$.

\section{4. - Le changement de fonction}

$$
\eta(z)=z^{-\frac{1}{2}+\mu} M(z)
$$

transforme l'équation différentielle (3) en l'équation confluente de WHittaker

$$
\frac{d^{2} M}{d z^{2}}-\left|\frac{1}{4}-\frac{1-4 \mu^{2}}{4 z^{2}}-\frac{k}{z}\right| M=o
$$

Des relations (4) et (13), on déduit que

$$
v(x, \lambda)=\psi(x) z^{-\frac{1}{2}}+\mu \cdot M(z) .
$$

Si $\mathcal{Y}\left[v_{1}, v_{2} ; x\right]$ désigne le wronskien des fonctions $v_{1}(x, \lambda), v_{2}(x, \lambda)$, on a

$$
\mathcal{W}\left[v_{1}, v_{2} ; x\right]=(2 \lambda)^{2} \mathcal{Q} \mathscr{M}\left[M_{1}, M_{2} ; z\right]
$$

et aux deux solutions linéairement indépendantes $v_{1}(x, \lambda), v_{2}(x, \lambda)$ de l'équation (2) correspondent les deux solutions linéairement indépendantes $M_{1}(z), M_{2}(z)$ de l'équation (14).

5. - Commençons par étudier le cas particulier $\nu=0$. On a $k=0, \mu=\frac{1}{2}$ et l'équation réduite s'écrit

$$
v^{\prime \prime}-\left\{\lambda^{2} g^{2}(x)+c(x)\right\} v=0
$$

avec

$$
c(x)=\frac{g^{\prime \prime}(x)}{2 g(x)}-\frac{3}{4} \frac{g^{\prime 2}(x)}{g^{2}(x)}
$$

L'équation de WHITraker correspondante est

$$
\frac{d^{2} M}{d z^{2}}-\frac{1}{4} M=0
$$

et a pour solutions linéairement indépendantes

$$
W_{0, \frac{1}{2}}(z) \equiv e^{z / 2}, W_{0, \frac{1}{2}}(-z) \equiv e^{-z / 2}
$$

Lorsque $\left(\rho-\frac{1}{2}\right) \pi \leq \arg z \leq\left(\rho+\frac{1}{2}\right) \pi$, $\rho$ entier, la solution générale de 
(18), $M(z)$ possède le comportement asymptotique $e^{\sigma z / 2} 0(1)\left({ }^{4}\right)$ avec $\sigma=(-1) \rho$. Puisque $\psi(x)$ est bornée dans l'intervalle $[\alpha, \beta]$, la solution générale de (17) est done

$$
v(x, \lambda)=e^{z / 2} 0(1)+e^{-z / 2} 0(1) .
$$

A $W_{0, \frac{1}{2}}(z)$, correspond la solution de (17)

$$
v_{1}(x, \lambda)=e^{\varepsilon / 2} 0(1)=0(1) e^{\lambda / g} g(s) d s
$$

à $W_{0, \frac{1}{2}}(-z)$ correspond la solution de (17)

$$
v_{2}(x, \lambda)=e^{-z / 20} 0(1)=0(1) e^{-\lambda \int_{0}^{x} g(s) d s} .
$$

Exemple. - La fonction $e^{x}$ vérifie les conditions imposées à $g(x)$ dans l'équation (17) quel que soit l'intervalle $[\alpha, \beta]$; l'équation (17) correspondante s'écrit

$$
v^{\prime \prime}-\left\{\lambda^{2} e^{2 x}+\frac{1}{4}\right\} v=0
$$

et a pour solutions linéairement indépendantes

$$
\begin{gathered}
v_{1}(x, \lambda)=e^{\lambda\left(e^{x}-1\right)} e^{-x / 2}, \\
v_{2}(x, \lambda)=e^{-\lambda\left(e^{x}-1\right)} e^{-x / 2} .
\end{gathered}
$$

6. - Dans le cas particulier, $b(x) \equiv 0$ ou $k=0(v \geq 1)$, M. R. LANGer a exprimé les solutions de l'équation (2) à l'aide des développements asymptotiques $\left(|\lambda| \geq \Lambda\right.$ ) des fonctions de Haxkes $[2,3, a]\left(^{5}\right)$. Ce résultat découle du fait que les changements de variable et de fonction $\eta=z^{\mu} y, z=2 i x$ transfor* ment l'equation (3) en l'équation de Bessel

$$
\frac{d^{2} y}{d x^{2}}+\frac{1}{x} \frac{d y}{d x}+\left(1-\frac{\mu^{2}}{x^{2}}\right) y=0
$$

On remarque, en outre que l'équation de WHITTAkme correspondant à ce cas particulier

$$
\frac{d^{2} M}{d z^{2}}-\left\lfloor\frac{1}{4}-\frac{1-4 \mu^{2}}{4 z^{2}}\right] M=0
$$

(4) La fonction désignée par 0(1) est uniformément bornée ainsi que sa dérivée par rapport à $z$ pour les valeurs de $z$ considérées.

(5) Les numéros entre crochets renvoient à la bibliographie. 
possède les solutions linéairement indépendantes

$$
\begin{aligned}
& W_{0, \mu}(z) \equiv \frac{1}{2}(\pi z)^{\frac{1}{2}} e^{\frac{i \pi}{2}(1+\mu)} H_{\mu *}^{(1)}\left(\frac{i z}{2}\right), \\
& W_{0, \mu}\left(z e^{-i \pi}\right) \equiv \frac{1}{2}(\pi z)^{\frac{1}{2}} e^{i \pi \mu / 2} H_{\mu}^{(2)}\left(\frac{i z}{2} e^{-i \pi}\right)
\end{aligned}
$$

où $H_{\mu}^{(1)}\left(\frac{i z}{2}\right)$ et $H_{\mu}^{(2)}\left(\frac{i z}{2} e^{-i \pi}\right)$ sont les fonctions de HANKEL.

\section{II. - Propriétés des solutions de l'équation réduite.}

7. - La solution générale de l'équation

$$
v^{\prime \prime}-\left[\lambda^{2} g^{2}(x)+b(x) \lambda+c(x)\right] v=0
$$

aveo $b \equiv-\frac{2 k g^{2}}{G}$ et $c \equiv \frac{\psi^{\prime \prime}}{\psi}$, s'écrit

$$
v\left(x, q_{2} \lambda\right)=\psi(x) z^{-\frac{1}{2}+\mu}\left[c_{1} M_{1}(z)+c_{2} M_{2}(z)\right]
$$

où $M_{1}(z)$ ef $M_{2}(z)$ désignent deux solutions linéairement indépendantes de l' equation (14).

Pour tontes les valeurs finies de la variable $z(|z| \leq M)$ et du module du paramètre complexe $\lambda$, nous prendrons comme solutions linéairement indépendantes $M_{1}(z), M_{2}(z)$ de (14), les fonctions $M_{k, \mu}(z)$ et $M_{k,-\mu}(z)$ exprimées respectivement par

$$
\begin{aligned}
& M_{1}(z) \equiv M_{k, \mu}(z)=z^{\frac{1}{2}+\mu \cdot e^{-z / 2}}\left[1+\frac{\frac{1}{2}+\mu-k}{2 \mu+1} z+\ldots\right] \\
& M_{2}(z) \equiv M_{k,-\mu}(z)=z^{\frac{1}{2}-\mu} e^{-z / 2}\left[1+\frac{\frac{1}{2}-\mu-k}{1-2 \mu} \cdot z+\ldots\right]
\end{aligned}
$$

$2 \mu$ est différent d'un entier $(\nu \neq 0)$ et les séries intervenant dans les relations (22) et (23) convergent uniformément pour les valeurs finies de $z$ et de $k(|z| \leq M)$.

Les fonctions $M_{k, \pm \mu}(z)$ sont uniformes si l'on convient comme au paragraphe 2 qu'une quantité élevée à une certaine puissance a son argument multiplió par cette puissance. De plus, elles vérifient les relations

$$
M_{k, \pm \mu}(z)=i \rho e^{ \pm i \pi \mu \rho} M_{\sigma k, \pm \mu}\left(z e^{-i \pi \rho)}\right.
$$


J. Lомвет-GoFfar: Etude des solutions d'équations différentielles, ete. 177

où $\rho$ désigne un entier et où $\sigma=(-1)^{p}$ et d'après [5], on a

$$
\mathcal{W}\left[M_{k, \mu}, M_{k,-\mu} ; z\right]=-2 \mu .
$$

Les solutions de l'equation (20) correspondant à $M_{k, \pm \mu}(z)$ s'écrivent

$$
v_{i}(z, \lambda)=\psi(x) z^{-\frac{1}{q}+\mu} M_{k, \pm \mu}(z), \quad i=1,2
$$

et d'après (16)

$$
\mathcal{W}\left[v_{1}, v_{2} ; x\right]=(-2 \mu)(2 \lambda)^{2 \mu}
$$

Puisque $|z| \leq M$, les solutions $v_{i}(x, \lambda)(i=1,2)$ sont uniformément bornées dans l'intervalle considéré.

8. - Lorsque $|z|>M$, on introduit les fonctions de Whitraker $W_{\sigma k, \mu}\left(e^{-i \pi_{\rho} z}\right)$ où $\rho$ et $\sigma$ ont été définis au paragraphe 7 .

Pour abréger l'écriture, on pose $[4, a]$

$$
W_{\sigma k, \mu}\left(e^{-i \pi \rho} z\right)=W_{\rho}(z)
$$

La fonction $W_{k, \mu}(z)$ est définie en fonction des $M_{k, \pm \mu}(z)$ par la relation [1]

$$
W_{k, \mu}(z)=\frac{\Gamma(-2 \mu)}{\Gamma\left(\frac{1-2 \mu}{2}-k\right)} M_{k, \mu}(z)+\frac{\Gamma(2 \mu)}{\Gamma\left(\frac{1+2 \mu}{2}-k\right)} M_{k,-\mu}(z) .
$$

De (24), il résulte done que

$$
W_{\rho}(z)=\frac{e^{-i \pi \mu \rho} \Gamma(-2 \mu)}{i \rho \Gamma\left(\frac{1-2 \mu}{2}-\sigma k\right)} M_{k, \mu}(z)+\frac{e^{i \pi \mu \rho \Gamma(2 \mu)}}{i \rho \Gamma\left(\frac{1+2 \mu}{2}-\sigma k\right)} M_{k,-\mu}(z)
$$

Si $\varepsilon$ désigne un nombre positif arbitrairement petit et si $|z|$ est suffisamment grand, pour les valeurs de arg $z$ situées dans le secteur

$$
\left(\rho-\frac{3}{2}\right) \pi+\varepsilon \leq \arg z \leq\left(\rho+\frac{3}{2}\right) \pi-\varepsilon,
$$

$W_{\rho}(z)$ possède le développement asymptotique

$$
W_{\rho}(z)=e^{-\frac{\sigma z}{2}}\left(z e^{-i \pi \rho}\right) \sigma k\left[1+\frac{0(1)}{z}\right]
$$


Dans le secteur

$$
\left(\rho-\frac{1}{2}\right) \pi+\varepsilon \leq \arg z \leq\left(\rho+\frac{3}{2}\right) \pi-\varepsilon, \quad|z|>M
$$

le développement asymptotique de $W_{\rho}(z)$ est fourni par la relation (32), celni de $W_{\rho+1}(z)$ s'obtient en remplaçant $\rho$ par $\rho+1$ dans le second membre de (32). Le wronskien de $W_{\rho}(z)$ et $W_{\rho+1}(z)$ est indépendant de $z$ et pent être évalué à partir des développements asymptotiques de ces fonctions; il vient

$$
\mathscr{W}\left[W_{\rho}, W_{\rho+1} ; z\right]=\sigma e^{i \pi \sigma k}
$$

d'où l'ón conclut que $W_{\rho}(z)$ et $W_{\rho+1}(z)$ sont linéairement indépendantes.

Dans le secteur $S_{\rho}(z)$ défini par

$$
\left(\rho-\frac{1}{2}\right) \pi \leq \arg z \leq\left(\rho+\frac{1}{2}\right) \pi \quad \text { si } \quad \mathfrak{R} e[\sigma k] \leq 0
$$

ou

$$
\left(\rho-\frac{1}{2}\right) \pi+\varepsilon \leq \arg z \leq\left(\rho+\frac{1}{2}\right) \pi-\varepsilon \quad \text { si } \quad \mathcal{R} e[\sigma k]>0
$$

la solution générale de l'équation de WHITTaker (14)

$$
c_{1} W_{p}(z)+c_{2} W_{p \pm 1}(z)\left({ }^{6}\right), \text { avec } c_{2} \neq 0
$$

est représentée asymptotiquement par

$$
c_{2} e^{\frac{1}{2} \sigma z}\left(z e^{-i \pi(\rho+1)}\right)-\sigma k 0(1)
$$

Dans ce secteur, $W_{\rho+1}(z)$ [resp. $\left.W_{\rho}(z)\right]$ tend vers l'infini [resp. zéro] lorsque $|z| \rightarrow \infty$, arg $z \neq(e \pm 1) \frac{\pi}{2}$.

Nous dirons que $W_{p+1}(z)$ est la solution dominante dans $S_{p}(z)$.

Il n'existe, par suite, dans $S_{\rho}(z)$, qu'une solution $W_{\rho}(z)$ possédant un đềveloppement asymptotique différent de $(34)$; toutes les autres solutions sont asymptotiquement identiques.

Dans $S_{p+1}(z)$, la solution dominante est $W_{\rho}(z)$.

Pour obtenir le développement asymptotique de $W_{\rho}(z)$ lorsque arg $z$ $W_{\rho-1}(z)$.

(6) Pour $\arg z \neq\left(\rho-\frac{1}{2}\right) \pi$, on prend la solution $W_{p+1}(z) ;$ si $\arg z=\left(\rho-\frac{1}{2}\right) \pi$, on utilise 
J. Loмвнт-GorFAR: Etude des solutions d'équations différentielles, etc. 179

n'appartient pas au secteur (31), on utilise les relations suivantes

$$
W_{k, \mu}\left(z e^{i \pi \rho}\right)=\frac{2 \pi i^{-1} e^{-i \pi k}}{\Gamma\left(\frac{1+2 \mu}{2}-k\right) \Gamma\left(\frac{1-2 \mu}{2}-k\right)} \frac{\sin \pi \mu}{\sin 2 \pi \mu} W_{-k, \mu}\left(z e^{-i \pi}\right)+
$$

$$
+\frac{i \rho}{\sin 2 \pi \mu} W_{k, \mu}(z)\left[\sin \pi \mu \rho e^{-2 i \pi k}+\sin \pi \mu(\rho+2)\right]
$$

lorsque $\rho$ est un entier pair et

$$
W_{k, \mu}\left(z e^{i \pi \rho}\right)=\frac{i^{\rho-1} W_{k, \mu}\left(\pi e^{-i \pi}\right)}{\sin 2 \pi \mu}\left[\sin \pi \mu(\rho+1) e^{2 i \pi k}+\sin \pi \mu(\rho-1)\right]+
$$

(36)

$$
+\frac{2 i \rho \pi e^{i \pi k}}{\Gamma\left(\frac{1+2 \mu}{2}-k\right) \Gamma\left(\frac{1-2 \mu}{2}-k\right)} W_{-k, \mu(\approx)} \frac{\sin \pi \mu(\rho+1)}{\sin 2 \pi \mu}
$$

lorsque $\rho$ est un entier impair.

9. - Pour obtenir le comportement asymptotique de $W_{\tau}(z)$ où $\tau=\rho+\nu+1$ dans $S_{p}(z)$, nous distinguerons deux cas.

i) y est impair. Si l'on remarque qu'à un nombre $\tau$ pair (resp. impair) correspond un nombre $\rho$ pair (resp. impair), on déduit de (30) que

$$
W_{\tau}(z)=K W_{p+1}(z)+K_{1} W_{p}(z)
$$

où

$$
K=\frac{2 \pi e^{-i \pi c k}}{i^{v} \sin 2 \pi \mu \Gamma\left(\frac{1-2 \mu}{2}-\sigma k\right) \Gamma\left(\frac{1+2 \mu}{2}-\sigma k\right)}
$$

$$
K_{1}=-i^{-\nu}\left[1-\frac{2 i e^{-i \pi \sigma k-i \pi \mu} \cos \pi(\sigma k-\mu)}{\sin 2 \pi \mu}\right]
$$

les constantes $K$ et $K_{1}$ sont finies ef $K$ est différent de zéro lorsque $k+\frac{1}{2} \pm v$ est différent d'un entier.

On a de même

$$
W_{\tau}(z)=K_{2} W_{p-1}(z)+K_{\mathrm{s}} W_{\rho}(z)
$$


où

$$
K_{2}=\frac{2 \pi e^{i \pi \sigma k}}{i^{\nu} \sin 2 \pi \mu \Gamma\left(\frac{1+2 \mu}{2}-\sigma k\right) \Gamma\left(\frac{1-2 \mu}{2}-\sigma k\right)}
$$

$$
K_{\mathrm{s}}=-i^{-, y}\left[1+2 i e^{i \pi \sigma k+i \pi \mu^{k}} \frac{\cos (\sigma k-\mu) \pi}{\sin 2 \pi \mu}\right]
$$

les constantes $K_{2}$ et $K_{3}$ sont finies et $K_{2}$ est différente de zéro lorsque $k+\frac{1}{2} \pm \mu$ n'est pas entier.

Des relations (37) et (39), il résulte que $W_{\tau}(z)$ et $W_{\rho}(z)$ sont linéairement indépendantes.

Ces relations fournissent en outre, une expression asymptotique de $W_{\tau}(z)$ valable dans le secteur $S_{\rho}(z)$. En effet, de (37), on déduit pour $|z|>M$ et

$$
\begin{gathered}
\left(\rho-\frac{1}{2}\right) \pi+\varepsilon \leq \arg z \leq\left(\rho-\frac{1}{2}\right) \pi \\
W_{\tau}(z)=\frac{2 \pi e^{i \pi \sigma k \rho}}{i^{\nu} \sin 2 \pi \mu \mathrm{r}\left(\frac{1-2 \mu}{2}-\sigma k\right) \Gamma\left(\frac{1+2 \mu}{2}-\sigma k\right)} e^{\frac{\sigma z}{2} z-\sigma k}\left[1+\frac{0(1)}{z}\right]
\end{gathered}
$$

lorsque $\mathscr{R} e(\sigma k)>0$, ce développement n'est valable que dans le secteur

$$
\left(\rho-\frac{1}{2}\right) \pi+\varepsilon \leq \arg z \leq\left(\rho+\frac{1}{2}\right) \pi-\varepsilon, \quad(|z|>M) .
$$

De (39), on conclut que la relation (41) est valable si $\mathscr{R} e(\sigma k)>0$, dans le secteur

$$
|z|>M, \quad\left(\rho-\frac{3}{2}\right) \pi+\varepsilon \leq \arg z \leq\left(\rho+\frac{1}{2}\right) \pi-\varepsilon,
$$

si $\mathscr{R} e(\sigma k) \leq 0$, dans le secteur

$$
|z|>M, \quad\left(\rho-\frac{3}{2}\right) \pi \leq \arg z \leq\left(\rho+\frac{1}{2}\right) \pi-\varepsilon .
$$

L'expression asymptotique (41) est done valable dans tont le secteur $S_{\rho}(z)$. ii). $\nu$ est pair. A un nombre $\rho$ pair correspond un entier $\tau+1$ pair et $a$ un nombre $\rho$ impair correspond un entier $\tau+1$ impair. On a donc d'après (30)

$$
W_{\tau+1}(z)=K_{4} W_{\rho+1}(z)+K_{5} W_{\rho}(z)
$$


où

$$
\begin{gathered}
K_{4}=\frac{\pi e^{-i \pi \sigma k}}{i^{\nu+1} \sin \pi \mu \Gamma\left(\frac{1+2 \mu}{2}-\sigma k\right) \Gamma\left(\frac{1-2 \mu}{2}-\sigma k\right)} \\
K_{5}=\frac{e^{i \pi \mu}}{i^{\nu+2}}\left[i-\frac{\pi e^{-i \pi \sigma k}}{\sin \pi \mu \Gamma\left(\frac{1+2 \mu}{2}+\sigma k\right) \Gamma\left(\frac{1-2 \mu}{2}-\sigma k\right)}\right]
\end{gathered}
$$

$K_{4}$ et $K_{5}$ sont des constantes finies et $K_{4}$ est différente de zéro si $k+\frac{1}{2} \pm \mu$ n'est pas entier.

On obtient de même, la relation

$$
W_{\tau+1}(z)=K_{\theta} W_{\rho-1}(z)+K_{7} W_{\rho}(z)
$$

où

$$
K_{6}=\frac{\pi e^{i \pi \sigma k}}{i^{\nu+1} \sin \pi \mu \Gamma\left(\frac{1+2 \mu}{2}-\sigma k\right) \Gamma\left(\frac{1-2 \mu}{2}-\sigma k\right)},
$$

$$
K_{7}=\frac{1}{i^{\nu+1}}\left|e^{i \pi \mu}-\frac{\pi i e^{-i \pi \mu} e^{i \pi \sigma k}}{\sin \pi \mu \Gamma\left(\frac{1+2 \mu}{2}+\sigma k\right) \Gamma\left(\frac{1-2 \mu}{2}-\sigma k\right)}\right|
$$

les constantes $K_{6}$ et $K_{\tau}$ sont finies et $K_{8}$ est différente de zéro si $k+\frac{1}{2} \pm \mu$ n'est pas entier.

On voit done, d'après $(42)$ et $(43)$ que $W_{\tau+1}(z)$ possède dans $S_{\rho}(z)$ le développement asymptotique

$$
W_{\tau+1}(z)=\frac{\pi e^{i \pi \sigma k_{p}}}{i^{\nu+1} \sin \pi \mu \Gamma\left(\frac{1+2 \mu}{2}-\sigma k\right) \Gamma\left(\frac{1-2 \mu}{2}-\sigma k\right)} e^{\sigma z} z^{-\sigma k} 0(1)
$$

10. - La solution de l'équation (21) correspondant a $W_{\rho}(z)$ s'écrit

$$
v_{\rho}(x, \lambda)=\psi(x) z^{\mu-\frac{1}{2}} W_{\rho}(z)
$$

D'après la relation (33), les solutions linéairement indépendantes de (14) dans les secteurs $S_{\rho}(z), \bmod . \frac{1}{\mu}\left(\rho=0, \ldots, \frac{1}{\mu}-1\right)$ sont les fonctions $W_{\rho}(z)$, $W_{\rho+1}(z)$ lorsque $\arg z \neq\left(\rho-\frac{1}{2}\right) \pi ;$ pour $\arg z=\left(\rho-\frac{1}{2}\right) \pi$ les deux solutions linéairement indépendantes de (14) sont $W_{\rho}(z), W_{\rho-1}(z)$. 
La relation (46) fait correspondre $v_{\rho}(x, \lambda), v_{\rho-1}(x, \lambda), v_{\rho+1}(x, \lambda)$ respectivement à $W_{\rho}(z), W_{\rho-1}(z), W_{\rho+1}(z)$.

De (30), (22), (23), il résulte que

$$
\begin{aligned}
& v_{\rho}(x, \lambda)=0(1), \\
& v_{\rho}^{\prime}(x, \lambda)=0\left(\lambda^{2 \mu}\right)
\end{aligned}
$$

pour $|z| \leq M,|\lambda| \geq \Lambda$.

Pour $|z|>M$ et

$$
\left(\rho-\frac{3}{2}\right) \pi+\varepsilon \leq \arg z \leq\left(\rho-\frac{3}{2}\right) \pi-\varepsilon,
$$

on a

$$
\begin{gathered}
v_{\rho}(x, \lambda)=e^{-\frac{1}{2} \sigma z z^{\sigma k+\mu-\frac{1}{2}} 0(1),} \\
v_{\rho}^{\prime}(x, \lambda)=e^{-\frac{1}{2} \sigma z} z^{\sigma k+\mu-\frac{1}{2}} 0(\lambda), \quad|\lambda| \geq \Lambda .
\end{gathered}
$$

Dans $S_{\rho}(z)$, les fonctions $v_{p}(x, \lambda)$ et $v_{\rho \pm 1}(x, \lambda)$ sont linéairement indépendantes puisque

$$
\mathcal{H}\left[v_{p}, v_{\rho \pm 1} ; x\right]=(2 \lambda)^{2}{ }^{2} \sigma e^{i \pi \sigma k} .
$$

Les fonctions $v_{\tau}(x, \lambda)$ et $v_{\rho}(x, \lambda)$ sont linéairement indépendantes si $k+\frac{1}{2} \pm \mu$ est différent d'un entier lorsque $\nu$ est impair. Lorsque $\vee$ est pair, les fonotions $v_{\tau+1}(x, \lambda)$ et $v_{\rho}(x, \lambda)$ sont linéairement indépendantes sous la même condition.

III. - Relation entre les solutions des équations (1) et (21)

11. - L'équation différentielle (21) peat s'écrire

$$
v^{\prime \prime}-\left\{\lambda^{2} g^{2}(x)-\omega(x, \lambda)\right\} v=0
$$

avec

$$
\omega(\boldsymbol{x}, \lambda)=\frac{k z^{\prime 2}(x, \lambda)}{z(x, \lambda)}-\frac{\psi^{\prime \prime}(x)}{\psi(x)}
$$

En outre, l'équation (1) est équivalente à

$$
u^{\prime \prime}-\left\{\lambda^{2} g^{2}(x)-\omega(x, \lambda)\right\} u=\theta(x, \lambda) u
$$


où

$$
\theta(x, \lambda)=\omega(x, \lambda)+\lambda b(x)+c(x)+X(x, \lambda)
$$

est une fonction continue de $\mathrm{x}$ dans $[\alpha, \beta]$ si les conditions des paragraphes 1 et 3 sont réalisées; de plus $\theta(x, \lambda)$ est uniformément bornée par rapport à $\lambda$ pour $|\lambda| \geq \Lambda$.

Soient $\vec{v}(x, \lambda)$ et $\vec{v}(x, \lambda)$ deux solutions linéairement indépendantes de l'équation (52), $v(x, \lambda)$ une solution quelconque de cette equation et $x_{0}$ un point de l'intervalle $[\alpha, \beta]$ et posons

$$
K(x, t, \lambda)=-\frac{\bar{v}(x, \lambda) \bar{v}(t, \lambda)-\bar{v}(x, \lambda) \bar{v}(t, \lambda)}{\mathcal{M}[\bar{v}, \bar{v} ; t]} \theta(t, \lambda) .
$$

Alors, la fonction $u(x, \lambda)$ définie par l'équation intégrale

$$
u(x, \lambda)=v(x, \lambda)+\int_{x_{0}}^{x} K(x, t, \lambda) u(t, \lambda) d t
$$

est une solution de l'équation différentielle (1) et puisque

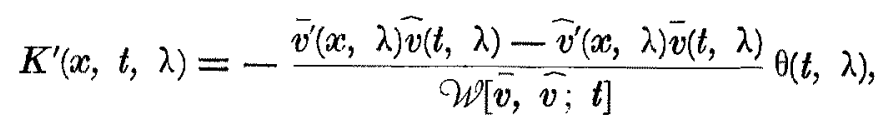

on a

$$
u^{\prime}(x, \lambda)=v^{\prime}(x, \lambda)+\int_{x_{0}}^{x} K^{\prime}(x, t, \lambda) u(t, \lambda) d t
$$

A la solution $v_{\rho}(x, \lambda)$ définie au paragraphe 10 , la relation (56) fait correspondre la solution $u_{\rho}, x_{0}(x, \lambda)$ de (54) qui se réduit à $v_{\rho}(x, \lambda)$ pour $x=x_{0}$ et dont la dérivée $u_{\rho}^{\prime}, x_{0}(x, \lambda)$ vaut $v_{\rho}^{\prime}(x, \lambda)$ en $x=x_{0}$.

On a done

$$
u_{p}, x_{0}\left(x_{0}, \lambda\right)=v_{p}\left(x_{0}, \lambda\right) \text { et } u_{\rho}^{\prime}, x_{0}\left(x_{0}, \lambda\right)=v_{\rho}^{\prime}\left(x_{0}, \lambda\right)
$$

Remarque. - Si $M$ désigne un nombre positif fixé assez grand, $|z(x, \lambda)|$ est inférieur à $M$ dans l'intervalle $\left[x_{-\Lambda}, x_{\Lambda}\right]$ de $[\alpha, \beta]$ qui contient l'origine et dont les extrémités varient avec $\lambda$. A l'extérieur de $\left[x_{-\Lambda}, x_{\Lambda}\right]$, on a $|z(x, \lambda)|>M$. 
IV. - Comportement asymptotique $(|\lambda| \geq \Lambda)$ de deux solutions linéairement indépendantes de (1) pour $x>0$.

12. - Rappelons d'abord un lemme [4(a), $4(b)]$.

Pour les valeurs de $\lambda$, telles $q u e|\lambda| \geq \Lambda$, on définit un intervalle $a_{1}(\lambda) \leq x \leq a_{2}(\lambda)$. Si $x, t$ et $x_{0}$ appartiennent à cet intervalle et si les fonctions $v(x, \lambda)$ et $K(x, t, \lambda)$ respectivement coefficient et noyau de l'équation intégrale (56) sont des fonctions continues des variables $x$ ef $t$, telles que

$$
\begin{aligned}
v(x, \lambda) & =0(1), \\
\int_{x_{0}}^{x}|K(x, t, \lambda)| d t & =0(\lambda-i), \quad j>0,
\end{aligned}
$$

alors, la solution $u_{x_{0}}(x, \lambda)$ de (56) égale à $v(x, \lambda)$ pour $x=x_{0}$, diffère de $v(x, \lambda)$ par une expression de l'ordre de $\lambda^{-j}$ et

$$
u_{x_{0}}(x, \lambda)=v(x, \lambda)+0(\lambda-j) \text {. }
$$

Ce lemme peut être modifié de la manière suivante: si les hypothèses (58) sont remplacées par

i).

$$
v(x, \lambda)=0(1) \text { ou } \sup _{x \in\left[a_{1}, a_{2}\right]}|v(x, \lambda)|=N_{i},
$$

ii). $K(x, t, \lambda)$ tend uniformément vers zéro pour $\lambda \rightarrow \infty$, la relation (59) devient

$$
u_{x_{0}}(x, \lambda)=v(x, \lambda)+0(\|K\|)
$$

où nous avons posé

$$
\|K\|=\sup _{x \in\left[a_{1}, a_{2}\right]} \int_{x_{0}}^{x}|K(x, t, \lambda)| d t .
$$

Pour démontrer la relation (60), on utilise le développement de Neumann de la fonction $u_{x_{0}}(x, \lambda)$

$$
u_{x_{0}}(x, \lambda)=v(x, \lambda)+K \text { ov }+K^{2} \text { ov }+\ldots+K^{(n+1)} \text { ov }+\cdots
$$

avec

$$
K^{(i)} \mathrm{ov}=\int_{x_{0}}^{x} K^{(i)}(x, t, \lambda) v(t, \lambda) d t .
$$


De l'inégalité

$$
\mid K^{(n)} \text { ov } \mid \leq\|K\|^{n} N_{1}
$$

on déduit que la série (61) majorée par la série uniformément convergente pour $|\lambda| \geq \Lambda$,

$$
N_{1}+\|K\| N_{1}+\ldots+\|K\|^{n} N_{1}+\ldots
$$

est uniformément convergente pour $|\lambda| \geq \Lambda$.

La quantité $u_{x_{0}}(x, \lambda)-v(x, \lambda)$ est done majorée par la série

$$
N_{1}\left[\|K\|+\|K\|^{2}+\ldots+\|K\|^{n}+\ldots\right]
$$

et l'on a

$$
u_{x_{0}}(x, \lambda)-v(x, \lambda)=0(\|K\|)
$$

Les fonctions

$$
\begin{aligned}
& \bar{v}(x, \lambda)=\psi(x) z^{-\frac{1}{2}+\mu} M_{k, \mu}(z), \\
& \bar{v}(x, \lambda)=\psi(x) z^{-\frac{1}{2}+\mu} M_{k,-\mu}(z)
\end{aligned}
$$

sont bornées pour $|z| \leq M$ et leur wronskien est égal à $(-2 \mu)(2 \lambda)^{2_{\mu}}$, (voir (27)).

Il en résulte puisque $\theta(t, \lambda)=0\left(\lambda^{-1}\right)$ que

$$
\int_{0}^{x}|K(x, t, \lambda)| d t=0(\lambda-4 \mu-1) \int_{z(0)}^{z(x)} z^{2 \mu-1} d z=0(\lambda-4 \mu-1)
$$

et d'après le lemme, on a

$$
u_{\rho, 0}(x, \lambda)=v_{\rho}(x, \lambda)+0\left(\lambda^{-4 \mu-1}\right), \quad \text { lorsque } \quad|z| \leq M
$$

Puisque $v_{\rho}^{\prime}(x, \lambda)=0\left(\lambda^{2 \mu}\right)$, il vient

$$
\boldsymbol{u}_{\rho, 0}^{\prime}(x, \lambda)=v_{\rho}^{\prime}(x, \lambda)+0\left(\lambda^{-2 \mu-1}\right), \text { lorsque }|z| \leq M .
$$

13. - Pour étudier le comportement des solutions de l'équation (1) pour les différentes valeurs du paramètre complexe $\lambda$, nous considérerons d'abord l'intervalle $[0, \beta]$.

Dans $S_{\rho}(\lambda)$, désignons par $\left.\bar{v}(x, \lambda), \widehat{v(x}, \lambda\right)$ les solutions linéairement indé- 
pendantes de l'équation (52) définies respectivement par

$$
\bar{v}(x, \lambda) \equiv v_{\rho}(x, \lambda)=\psi(x) z^{-\frac{1}{2}+\mu} W_{\rho}(z)
$$

$$
\bar{v}(x, \lambda) \equiv \begin{cases}v_{\rho+1}(x, \lambda)=\psi(x) z^{-\frac{1}{2}+\mu} W_{\rho+1}(z) & \text { pour arg } z \neq\left(\rho-\frac{1}{2}\right) \pi \\ v_{p-1}(x, \lambda)=\psi(x) z^{-\frac{1}{2}+\mu} W_{p-1}(z) & \text { pour arg } \mathrm{z}=\left(\rho-\frac{1}{2}\right) \pi\end{cases}
$$

soit $u_{\rho, \beta}(x, \lambda)$ la solution de l'équation intégrale (56) qui se réduit à $v(x, \lambda) \equiv$ $\equiv v_{\rho}(x, \lambda)$ pour $x=\beta$.

Si l'on pose

$$
\begin{aligned}
& \Delta\left(v_{\rho}, v_{\rho \pm 1}\right)=v_{\rho}(x) v_{\rho \pm 1}(t)-v_{\rho}(t) v_{\rho \pm 1}(x),
\end{aligned}
$$

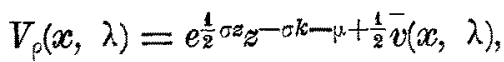

$$
\begin{aligned}
& V_{p \pm 1}(x, \lambda)=e^{-\frac{1}{2} \sigma z^{\sigma} k-\mu+\frac{1}{2}} \widehat{v}(x, \lambda), \\
& U_{\rho, \beta}(x, \lambda)=e^{\frac{1}{2} \sigma z_{z}-\sigma k-\mu+\frac{1}{2}} u_{\beta, \beta}(x, \lambda)
\end{aligned}
$$

les fonctions $V_{\rho}(x, \lambda)$ et $V_{\rho \pm 1}(x, \lambda)$ sont $0(1)$ pour $|z|>M$ et de l'équation intégrale $(56)$, il résulte que

$$
U_{\rho, \beta}(x, \lambda)=V_{\rho}(x, \lambda)+0(\lambda-2 \mu-1) \int_{x}^{\beta} z(t)^{2 \mu-1} U_{\rho, \beta}(t, \lambda) d t
$$

puisque pour $t>x$ dans l'intervalle $\left[x_{\Lambda}, \beta\right], \arg [z(t)-z(x)]=\arg \lambda$ et $e^{\sigma[z(x)-z(t)]}$ est bornée. Toutefois, lorsque $\mathscr{R} e \sigma k>0$, la quantité $\left[\frac{z(x)}{z(t)}\right]^{-2 \sigma k}{ }^{2}$ 'est pas bornée pour les petites valeurs de $\frac{z(x)}{z(t)}$. Mais si $\frac{z(x)}{z(t)}=\delta,(\delta>0)$, on a

$$
\left|e^{\sigma[z(x)-z(t)]}\right| \leq e^{M\left(1-\frac{1}{\delta}\right) \sin \varepsilon}
$$

et puisque $e^{-M \sin s / \delta \delta-2 \sigma k}$ est bornée quel que soit $\delta>0$ et tend vers zéro si $\delta \rightarrow 0$, l'expression $e^{\sigma[z(x)-z(t)]}\left[\frac{z(x)}{z(t)}\right]^{-2 \sigma k}$ est bornée pour les valeurs de $x, t, z$
considérées. En appliquant le lemme du paragraphe 12 à l'équation intégrale (66), on 
roit que

$$
\begin{aligned}
& U_{\beta, \beta}(x, \lambda)=V_{\rho}(x, \lambda)+0\left(\lambda^{-2}\right) \text { pour } \mu \neq \frac{1}{4},|z|>M, \\
& U_{\rho, \beta}(x, \lambda)=V_{\rho}(x, \lambda)+0\left(\lambda^{-2} \lg \lambda\right) \text { pour } \mu=\frac{1}{4},|z|>\mathrm{M} .
\end{aligned}
$$

On en déduit

$$
\begin{aligned}
& u_{\rho, \beta}(x, \lambda)=v_{\rho}(x, \lambda)+e^{-\frac{1}{2} \sigma z} z^{\sigma k+\mu-\frac{1}{2}} 0(\lambda-2) \text { pour } \mu+\frac{1}{4},|z|>M \\
& \left.u_{\rho, \beta}(x, \lambda)=v_{\rho}(x, \lambda)+e^{-\frac{1}{2} \sigma z z^{\sigma} k+\mu-\frac{1}{2} 0(\lambda-2} \lg \lambda\right) \text { pour } \mu=\frac{1}{4},|z|>M .
\end{aligned}
$$

D'après les relations (50), (57), on obtient

$$
\begin{aligned}
& u_{\rho, \beta}^{\prime}(x, \lambda)=v_{\rho}^{\prime}(x, \lambda)+e^{-\frac{1}{2} \sigma z \sigma k} \tilde{g}^{\sigma}+\mu-\frac{1}{2} O(\lambda-1), \quad \text { pour } \quad \mu \neq \frac{1}{4},|z|>M, \\
& u_{\rho, \beta}^{\prime}(x, \lambda)=v_{\rho}^{\prime}(x, \lambda)+e^{-\frac{1}{2} \sigma z^{\sigma} k+\mu-\frac{1}{2}} 0\left(\lambda^{-1} \lg \lambda\right), \quad \text { pour } \quad \mu=\frac{1}{4},|z|>M .
\end{aligned}
$$

14. - Lorsque $x \in\left[0, x_{\Lambda}\right]$, on a $|z(x)| \leq M$ et l'équation intégrale (56) s'écrit

$$
u_{\rho, \beta}(x, \lambda)=v_{\rho}(x, \lambda)+I_{1}+I_{2}
$$

si l'on désigne respectivement par $I_{1}, I_{2}$ les deux intégrales

$$
\begin{aligned}
& I_{1}=\int_{x_{\Lambda}}^{\beta} \frac{\Delta\left(v_{\rho}, v_{\rho \pm 1}\right) \theta(t, \lambda)}{\mathcal{U}\left[v_{\rho}, v_{\rho \pm 1} ; t\right]} u_{\rho, \beta}(t, \lambda) d t \\
& I_{2}=\int_{x}^{x_{\Lambda}} \frac{\Delta\left(v_{\rho}, v_{\rho \pm 1} \theta(t, \lambda)\right.}{\mu_{\rho}\left[v_{\rho}, v_{\rho \pm 1} ; t\right]} u_{\rho, \beta}(t, \lambda) d t
\end{aligned}
$$

Puisque $v_{\rho}(x, \lambda)$ et $v_{\rho \pm 1}(x, \lambda)$ sont bornées dans l'intervalle $\left[0, x_{\Lambda,}\right.$, on a d'après $(67),(68)$ (dans $I_{1}$, on sait que $t \in\left[x_{\Lambda}, \beta\right]$

$$
I_{1}=\left\{\begin{array}{l}
O\left(\lambda^{-2} \lg \lambda\right) \text { pour } \mu=\frac{1}{4}, \\
O\left(\lambda^{-2}\right) \text { pour } \mu \neq \frac{1}{4}
\end{array}\right.
$$


Pour $x \in\left[0, x_{\Lambda}\right]$, [resp. $t \in\left[x, x_{\Lambda}\right]$, les fonctions $v_{\rho}(x, \lambda), v_{p \pm 1}(x, \lambda)$ [resp. $\left.v_{p}(t, \lambda), v_{p \pm 1}(t, \lambda)\right]$ sont bornées et l'on a

$$
I_{2}=0(\lambda-4 \mu-1)
$$

Les hypothèses du lemme du paragraphe 12 sont done vérifiées pour l'équation intégrale

$$
u_{\rho, \beta}(x, \lambda)=f(x, \lambda)+I_{2}
$$

avec

$$
f(x, \lambda) \equiv v_{p}(x, \lambda)+I_{1}=0(1)
$$

On en conclut

$$
\begin{aligned}
& u_{\rho, \beta}(x, \lambda)=v_{\rho}(x, \lambda)+0\left(\lambda^{-2} \lg \lambda\right), \text { pour } \mu=\frac{1}{4},|z| \leq M, \\
& u_{\rho, \beta}(x, \lambda)=v_{\rho}(x, \lambda)+0(\lambda-4 \mu-1), \text { pour } \mu \neq \frac{1}{4},|z| \leq M .
\end{aligned}
$$

En procédant de la même manière, on obtient pour la dérivée $u_{\rho, \beta}^{\prime}(x, \lambda)$

$$
\begin{aligned}
& u_{\rho, \beta}^{\prime}(x, \lambda)=v_{\rho}^{\prime}(x, \lambda)+0(\lambda-3 / 2 \lg \lambda), \text { pour } \mu=\frac{1}{4},|z| \leq M, \\
& u_{\rho, \beta}^{\prime}(x, \lambda)=v_{\rho}^{\prime}(x, \lambda)+0\left(\lambda^{-2 \mu-1}\right), \text { pour } \mu \neq \frac{1}{4},|z| \leq M .
\end{aligned}
$$

15. - Recherchons maintenant le comportement des fonctions $u_{\rho \pm 1,0}(x, \lambda)$ dans $S_{p}(\lambda)$, pour les différentes valeurs du paramètre complexe $\lambda$; comme nous le verrons ci-dessous, $u_{\rho, \beta}(x, \lambda)$ et $u_{\rho \pm 1,0}(x, \lambda)$ sont deux solutions de l'équation (1) linéairement indépendantes dans $S_{\rho}(\lambda)$.

Remarquons d'abord que les fonctions $u_{p \pm 1,0}(x, \lambda)\left({ }^{7}\right)$ sont les solutions de l'équation intégrale $(56)$ où $x_{0}=0$ et $v(x, \lambda) \equiv v_{\rho \pm 1}(x, \lambda)$.

Lorsque $x \in\left[0, x_{\Lambda}\right]$, le comportement de $u_{p \pm 1,0}(x, \lambda)$ et de sa dérivée $u_{p \pm 1,0}^{\prime}(x, \lambda)$ s'obtient comme au paragraphe $12 ;$ on a

$$
\begin{array}{lll}
u_{p \pm 1,0}(x, \lambda)=v_{\rho \pm 1}(x, \lambda)+0\left(\lambda^{-4 \mu-1}\right), & \text { pour } & |z| \leq M, \\
u_{p \pm 1,0}^{\prime}(x, \lambda)=v_{\rho \pm 1}^{\prime}(x, \lambda)+0\left(\lambda^{-2 \mu-1}\right), & \text { pour } \quad|z| \leq M .
\end{array}
$$

$(7)$ On utilise les solutions $u_{\rho-1,},(x, \lambda)$ et $v_{\beta-1}(x, \lambda)$ lorsque $\arg z=\left(\rho-\frac{1}{2}\right) \pi$, pour
${ }_{k} \leq 0$. Re ok $\leq 0$. 
J. LonbeT-GoFfar: Etude des solutions d'équations différentielles, etc. 189

Si $x \in\left[x_{\Lambda}, \beta\right]$, l'équation intégrale (56) s'écrit

$$
u_{p \pm 1,0}(x, \lambda)=v_{p \pm 1}(x, \lambda)-I_{3}-I_{4}
$$

avec

$$
\begin{aligned}
& I_{3}=\int_{0}^{x_{\Lambda}} \frac{\Delta\left(v_{\rho}, v_{\rho \pm 1}\right) \theta(t, \lambda)}{\mathcal{W}\left[v_{\rho}, v_{\rho \pm 1} ; t\right]} u_{\rho \pm 1,0}(t, \lambda) d t \\
& I_{5}=\int_{x_{\Lambda}}^{x} \frac{\Delta\left(v_{\rho}, v_{\rho \pm 1}\right) \theta(t, \lambda)}{\mathcal{W}\left[v_{\rho}, v_{\rho \pm 1} ; t\right]} u_{\rho \pm 1,0}(t, \lambda) d t .
\end{aligned}
$$

La relation (75) donne le comportement de la fonction $u_{\rho \pm 1,0}(t, \lambda)$ de $I_{3}$ et il vient

$$
I_{3}=e^{\frac{1}{2} \sigma z(x)} z(x)^{-\alpha z+\mu-\frac{1}{2}} 0(\lambda-4 \mu-1)
$$

puisque seule la fonction $v_{\rho \pm 1}(x, \lambda)$ de $I_{3}$ n'est pas bornée.

En posant

$$
U_{\rho \pm 1,0}(x, \lambda)=e^{-\frac{1}{2} \sigma z(x)} z(x)^{\sigma k+\frac{1}{2}-p} \boldsymbol{u}_{\rho \pm 1,0}(\boldsymbol{x}, \lambda),
$$

nous voyons que

$$
I_{4} e^{-\frac{1}{2} \sigma z(x)} z(x)^{\sigma k-\mu+\frac{1}{2}}=0(\lambda-2 \mu-1) \int_{x_{\Lambda}}^{x} z(t)^{2 \mu-1} U_{\beta \pm 1, o}(t, \lambda) d t
$$

car la fonction $e^{-\sigma[z(x)-z(t)]}\left[\frac{z(x)}{z(t)}\right]^{2 \sigma k}$ est bornée pour $t<x$.

Le noyau $K(x, t, \lambda)$ de l'équation intégrale

$$
U_{\rho \pm 1,0}(x, \lambda)=F(x, \lambda)-e^{-\frac{1}{2} \sigma z(x)} z(x)^{\sigma k-\mu+\frac{1}{2}} I_{4}
$$

avec

$$
\begin{aligned}
F(x, \lambda) & \equiv V_{\rho \pm 1}(x, \lambda)-e^{-\frac{1}{2} \sigma z(x) z(x)^{\sigma k-\mu+\frac{1}{2}} I_{3}} \\
& =0(1)
\end{aligned}
$$

vérifie la relation

$$
\int_{x_{\Lambda}}^{x}|K(x, t, \lambda)| d t=\left\{\begin{array}{lll}
0\left(\lambda^{-2} \lg \lambda\right) & \text { si } \mu=\frac{1}{4} \\
0\left(\lambda^{-2}\right) & \text { si } & \mu \neq \frac{1}{4}
\end{array}\right.
$$


du lemme du paragraphe 12, on déduit alors

$$
\begin{aligned}
& U_{\rho \pm 1,0}(x, \lambda)=V_{\rho \pm 1}(x, \lambda)+0\left(\lambda^{-2} \lg \lambda\right) \text { pour } \mu=\frac{1}{4},|z|>M, \\
& U_{\rho \pm 1,0}(x, \lambda)=V_{\rho \pm 1}(x, \lambda)+0\left(\lambda^{-4 \mu-1}\right) \text { pour } \mu \neq \frac{1}{4},|z|>M .
\end{aligned}
$$

Il en résulte que

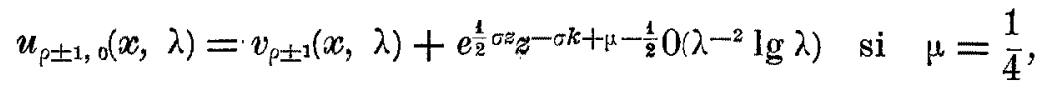

$$
\begin{aligned}
& u_{\rho \pm 1,0}(x, \lambda)=v_{\rho \pm 1}(x, \lambda)+e^{\frac{1}{2} \sigma z} z^{-\alpha k+\mu-\frac{1}{2}} 0(\lambda-4 \mu-1) \quad \text { si } \quad \mu \neq \frac{1}{4}, \quad|z|>M .
\end{aligned}
$$

Pour la dérivée $u_{\rho \pm 1,0}^{\prime}(x, \lambda)$ on obtient

$$
\begin{gathered}
\left.u_{\rho \pm 1,0}^{\prime}(x, \lambda)=v_{\rho \pm 1}^{\prime}(x, \lambda)+e^{\frac{1}{2} \sigma z z-\sigma k+\mu-\frac{1}{2} 0(\lambda-1} \lg \lambda\right) \text { si } \mu=\frac{1}{4}, \\
u_{\rho \pm 1,0}^{\prime}(x, \lambda)=v_{\rho \pm 1}^{\prime}(x, \lambda)+e^{\frac{1}{2} \sigma z} z^{-\sigma k+\mu-\frac{1}{2} 0(\lambda-4 \mu)} \text { si } \mu \neq \frac{1}{4}, \quad|z|>M .
\end{gathered}
$$

16. - Il reste à démontrer que les solutions $u_{p \pm 1,0}(x, \lambda)$ et $u_{p, p}(x, \lambda)$ de l'équation differentielle (1) sont linéairement indépendantes. Le wronskien des deux solutions $u_{\rho+1,0}(x, \lambda)\left[\right.$ ou $u_{\rho-1,0}(x, \lambda)$ si $\left.\arg z=\left(\rho-\frac{1}{2}\right) \pi\right]$ et $u_{\rho, p}(x, \lambda)$ est indépendant de $x$ et peut être évalué à l'aide des relations (75), (76) et (71), (72), (73), (74).

Pour $|\lambda| \geq \Lambda, \arg \lambda=\arg z \in S_{p}(\lambda)$, il vient

$$
\begin{aligned}
& \mathcal{U}\left[u_{\rho, \beta}, u_{\rho \pm 1,0}\right]=\mathcal{U}\left[v_{\rho}, v_{\rho \pm 1}\right]+0\left(\lambda^{-3 / 2} \lg \lambda\right) \text { pour } \mu=\frac{1}{4}, \\
& \mathcal{U}\left[u_{\rho, \beta}, u_{\rho \pm 1,0}\right]=\mathcal{W}\left[v_{\rho}, v_{\rho \pm 1}\right]+0\left(\lambda^{-2 \mu-1}\right) \text { pour } \mu \neq \frac{1}{4} .
\end{aligned}
$$

Le tableau suivant, fournit les solutions linéairement indépendantes de (1) correspondant à $0 \leq \arg \lambda \leq 2 \pi, x>0$ 
J. LombeT-Gorfar: Etude des solutions d'équations différentielles, etc. 191

valeurs de $\lambda$

Solutions linéairement indépendantes

i). $\mathscr{R} e \sigma k>0$

$\begin{array}{lll}{\left[0, \frac{\pi}{2}\right]} & u_{0, \beta}(x, \lambda) & u_{1,0}(x, \lambda) \\ {\left[\frac{\pi}{2}, \frac{3 \pi}{2}[\right.} & u_{1, \beta}(x, \lambda) & u_{0,0}(x, \lambda) \\ {\left[\frac{3 \pi}{2}, 2 \pi\right]} & u_{2, \beta}(x, \lambda) & u_{1,0}(x, \lambda)\end{array}$

ii). $\mathscr{R} e \sigma k \leq 0$

$$
\begin{array}{lll}
{\left[0, \frac{\pi}{2}\right]} & u_{0, \beta}(x, \lambda) & u_{1,0}(x, \lambda) \\
{\left[\frac{\pi}{2}, \frac{3 \pi}{2}\right]} & u_{1, \beta}(x, \lambda) & u_{0,0}(x, \lambda) \\
{\left[\frac{3 \pi}{2}, 2 \pi\right]} & u_{2, \beta}(x, \lambda) & u_{1,0}(x, \lambda)
\end{array}
$$

V. - Comportement asymptotique de deux solutions linéairement independantes de (1) pour $x<0$.

17. - Nous étudierons maintenant le comportement des solutions de l'équation (1) lorsque $x$ est négatif, $x \geq \alpha$; dans ce cas, on a

$$
\arg z=\arg \lambda+\pi(\nu+1) \text {. }
$$

D'importantes modifications s'introduisent ioi.

Remarquons d'abord que la solution $v_{\tau}(x, \lambda)$ ( $\tau$ entier) de l'óquation (21) tend vers zéro lorsque $|z| \rightarrow \infty$ et $R e \sigma k \leq 0$, dans le secteur

identique à

$$
\pi\left(\tau-v-\frac{3}{2}\right) \leq \arg \lambda \leq \pi\left(\tau-v-\frac{1}{2}\right)
$$

$$
\pi\left(\rho-\frac{1}{2}\right) \leq \arg \lambda \leq \pi\left(\rho+\frac{1}{2}\right)
$$

si $\tau=\rho+v+1$.

Lorsque Reøk>0, ce secteur se réduit à

$$
\pi\left(\tau-\nu-\frac{3}{2}\right)+\varepsilon \leq \arg \lambda \leq \pi\left(\tau-\nu-\frac{1}{2}\right)-\varepsilon .
$$


A un entier $\vee$ impair, correspond un entier $\tau$ pair (resp. impair) si $\rho$ est pair (resp. impair) mais à un entiex $\vee$ pair correspond un entier $\tau$ impair (resp. pair) si $\rho$ est pair (resp. impair). On a donc

$$
\begin{aligned}
& v_{\tau}(x, \lambda)=e^{-\sigma z(x) / 2} z(x)^{\sigma k+\mu-\frac{1}{2}} 0(1), \text { pour } \quad \vee \text { impair } \\
& v_{\tau}(x, \lambda)=e^{\sigma \varepsilon(x) / 2} z(x)^{-\sigma k+\mu-\frac{1}{2}} 0(1), \text { pour } \vee \vee \text { pair. }
\end{aligned}
$$

Les résultats relatifs au cas où $\vee$ est pair se déduisent de ceux que nous allons obtenir pour $\nu$ impair, en permutant les rôles des indices $\tau$ et $\tau \pm 1$.

Supposons done $\vee$ impair et $x \in\left[\alpha, x_{-\Lambda}\right]$ c'est-à-dire $|z|>M$.

La solution $u_{\tau, \alpha}(x, \lambda)$ de l'équation différentielle (1) vérifie l'équation intégrale

$$
u_{\tau, \alpha}(x, \lambda)=v_{\tau}(x, \lambda)-\int_{\alpha}^{x} \frac{\Delta\left(v_{\tau}, v_{\tau \pm 1}\right) \theta(t, \lambda)}{\mathcal{W}\left[v_{\tau}, v_{\tau \pm 1} ; t\right]} u_{\tau, \alpha}(t, \lambda) d t
$$

Si l'on introduit dans l'équation intégrale (84) la formule (47), la définition de $\left(\Delta v_{\tau}, v_{\tau \pm 1}\right)$ et les relations

$$
\begin{aligned}
& V_{\tau}(x, \lambda)=e^{\sigma z(x) / 2} z(x)-\sigma k-\mu+\frac{1}{2} v_{\tau}(x, \lambda),
\end{aligned}
$$

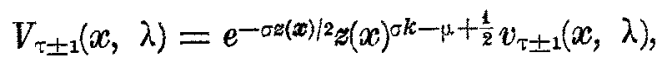

$$
\begin{aligned}
& U_{\tau, \alpha}(x, \lambda)=e^{\sigma z(x) / 2} z(x)^{-\sigma k-\mu+\frac{1}{2}} u_{\tau, \alpha}(x, \lambda)
\end{aligned}
$$

on voit que

$$
U_{\tau, \alpha}(x, \lambda)=V_{\tau}(x, \lambda)+0\left(\lambda^{-2 \mu-1}\right) \int_{\alpha}^{x} z(t)^{2 \mu-1} U_{\tau, \alpha}(t, \lambda) d t
$$

puisque la fonction $e^{\sigma[z(x)-z(t)]}\left[\frac{z(x)}{z(t)}\right]^{-2 \sigma k}$ est bornée pour $t<x(|t|>|x|)$, arg $\lambda \in S_{p}(\lambda)$.

Il en résulte d'après le lemme du paragraphe 12 ,

$$
\begin{aligned}
& U_{\tau, \alpha}(x, \lambda)=V_{\tau}(x, \lambda)+0\left(\lambda^{-2} \lg \lambda\right) \text { si } \mu=\frac{1}{4}, \\
& U_{\tau, \alpha}(x, \lambda)=V_{\tau}(x, \lambda)+0\left(\lambda^{-2}\right) \text { si } \mu \neq \frac{1}{4} .
\end{aligned}
$$


Il vient finalement

$$
\left.u_{\tau, \alpha}(x, \lambda)=v_{\tau}(x, \lambda)+e^{-\sigma z(x) / 2 z(x)^{\sigma k+\mu-\frac{1}{2}} 0(\lambda-2} \lg \lambda\right) \quad \text { si } \quad \mu=\frac{1}{4},
$$

(86) $\quad u_{\tau, \alpha}(x, \lambda)=v_{\tau}(x, \lambda)+e^{-\sigma z(x) / 2} \approx(x)^{\sigma k+\mu-\frac{1}{2}} 0(\lambda-2) \quad$ si $\quad \mu \neq \frac{1}{4}, \quad|z|>M$.

Pour la dérivée $u_{\tau, \alpha}^{\prime}(x, \lambda)$, on obtient

$$
\begin{aligned}
& \left.u_{\tau, \alpha}^{\prime}(x, \lambda)=v_{\tau}^{\prime}(x, \lambda)+e^{-\sigma(x) / 2} z(x)^{\sigma k+\mu-\frac{1}{2}} O(\lambda-1) \lg \lambda\right) \quad \text { si } \quad \mu=\frac{1}{4}, \quad|z|>M, \\
& u_{\tau, \alpha}^{\prime}(x, \lambda)=v_{\tau}^{\prime}(x, \lambda)+e^{-\sigma z(x) / 2} z(x)^{\sigma k+\mu-\frac{1}{2} O(\lambda-1)} \text { si } \mu \neq \frac{1}{4}, \quad|z|>M .
\end{aligned}
$$

18. - Lorsque $x \in\left[x_{-\Lambda}, 0\right],|z(x)| \leq M$, l'équation intégrale (56) devient

$$
u_{\tau, \alpha}(x, \lambda)=v_{\tau}(x, \lambda)-I_{5}-I_{6}
$$

avec

$$
\begin{aligned}
& I_{5}=\int_{\alpha}^{x_{-\Lambda}} \frac{\Delta\left(v_{\tau}, v_{\tau \pm 1}\right) \theta(t, \lambda)}{\mathcal{W}\left[v_{\tau}, v_{\tau \pm 1} ; t\right]} u_{\tau, \alpha}(t, \lambda) d t \\
& I_{6}=\int_{x_{-\Lambda}}^{x} \frac{\Delta\left(v_{\tau}, v_{\tau \pm 1}\right) \theta(t, \lambda)}{\mathcal{W}\left[v_{\tau}, v_{\tau \pm 1} ; t\right]} u_{\tau, \alpha}(t, \lambda) d t .
\end{aligned}
$$

La fonction $u_{\tau, \alpha}(t, \lambda)$ de l'intégrale $I_{5}$ est donnée par les relations (85), (86) et puisque $v_{r}(x, \lambda), v_{\tau \pm 1}(x, \lambda)$ sont bornées pour $x \in\left[\alpha, x_{-\Lambda}\right]$, on a

$$
I_{5}= \begin{cases}o\left(\lambda^{-2} \lg \lambda\right) & \text { pour } \mu=\frac{1}{4}, \\ 0\left(\lambda^{-2}\right) & \text { pour } \mu \neq \frac{1}{4} .\end{cases}
$$

Les fonctions $v_{\tau}(x, \lambda), v_{\tau \pm \lambda}(x, \lambda), v_{\tau}(t, \lambda), v_{\tau \pm 1}(t, \lambda)$ de $I_{6}$ sont bornées et pour l'équation intégrale

$$
u_{\tau, \alpha}(x, \lambda)=f_{1}(x, \lambda)-I_{6}
$$

avec

$$
f_{1}(x, \lambda) \equiv v_{\tau}(x, \lambda)-I_{5}=0(1),
$$

les hypothèses du lemme du paragraphe 12, sont vérifiées. 
Il vient, par suite

$$
\begin{aligned}
& \boldsymbol{u}_{\tau, \alpha}(x, \lambda)=v_{\tau}(x, \lambda)+0\left(\lambda^{-2} \lg \lambda\right), \quad \text { si } \quad \mu=\frac{1}{4}, \quad|\boldsymbol{z}| \leq M, \\
& \boldsymbol{u}_{\tau, \alpha}(x, \lambda)=v_{\tau}(x, \lambda)+0\left(\lambda^{-4 \mu-1}\right), \quad \text { si } \quad \mu \neq \frac{1}{4}, \quad|z| \leq M .
\end{aligned}
$$

Le même procédé appliqué à la dérivée $u_{\tau, \alpha}^{\prime}(x, \lambda)$ donne

$$
\begin{aligned}
& u_{\tau, \alpha}^{\prime}(x, \lambda)=v_{\tau}^{\prime}(x, \lambda)+0\left(\lambda^{-3 / 2} \lg \lambda\right), \quad \text { si } \mu=\frac{1}{4}, \quad|z| \leq M, \\
& u_{\tau, \alpha}^{\prime}(x, \lambda)=v_{\tau}^{\prime}(x, \lambda)+0\left(\lambda^{-2 \mu-1}\right), \quad \text { si } \quad \mu \neq \frac{1}{4}, \quad|z| \leq M .
\end{aligned}
$$

19. - La seconde solution de (1) correspondant an cas où $x$ est négatif et où $v$ est un entier positif impair est la fonction $u_{\tau \pm 1,0}(x, \lambda)$. En procédant comme an paragraphe 15 , on obtient

$$
\begin{aligned}
& u_{\tau \pm 1,0}(x, \lambda)=v_{\tau \pm 1}(x, \lambda)+0(\lambda-4 \mu-1), \text { si }|z| \leq M, \\
& u_{\tau \pm 1,0}^{\prime}(x, \lambda)=v_{\tau \pm 1}^{\prime}(x, \lambda)+0\left(\lambda^{-2 \mu-1}\right), \quad \text { si } \quad|z| \leq M,
\end{aligned}
$$

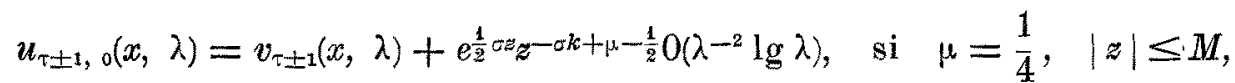

$$
\begin{aligned}
& u_{\tau \pm 1}, 0(x, \lambda)=v_{\tau \pm 1}(x, \lambda)+e_{\frac{1}{2} \sigma z}-\sigma k+\mu-f_{2}^{\frac{1}{2}} 0(\lambda-4 \mu-1), \quad \text { si } \mu \neq \frac{1}{4}, \quad|z| \leq M \\
& \left.\boldsymbol{u}_{\tau \pm 1,0}^{\prime}(x, \lambda)=v_{\tau \pm 1}^{\prime}(x, \lambda)+e^{\frac{1}{2} \sigma z} z^{-\sigma k+\mu-\frac{1}{2} 0(\lambda-1} \lg \lambda\right), \quad \text { si } \quad \mu=\frac{1}{4}, \quad|z| \leq M, \\
& u_{\tau \pm 1,0}^{\prime}(x, \lambda)=v_{\tau \pm 1}^{\prime}(x, \lambda)+e^{\frac{1}{2} \sigma z_{z}-\sigma k+\mu-\frac{1}{2} O(\lambda-4 \mu),} \text { si } \quad \mu \neq \frac{1}{4}, \quad|z| \leq M
\end{aligned}
$$

Les solutions $u_{\tau \pm 1,0}(x, \lambda)$ et $u_{\tau, a}(x, \lambda)\left[\right.$ ou $u_{\tau-1, o}(x, \lambda)$ et $u_{\tau, a}(x, \lambda)$ lorsque $\left.\arg z=\left(\tau-\frac{1}{2}\right) \pi\right]$ sont linéairement indépendantes puisque

$$
\begin{aligned}
& \mathcal{W}\left[u_{\tau \pm 1,0} ; u_{\tau, \alpha}\right]=\mathcal{W}\left[v_{\tau \pm 1}, v_{\tau}\right]+O\left(\lambda^{-3 / 2} \lg \lambda\right) \text { pour } \mu=\frac{1}{4}, \\
& \mathcal{W}\left[u_{\tau \pm 1,0} ; u_{\tau, \alpha}\right]=\mathcal{W}\left[v_{\tau \pm 1}, v_{\tau}\right]+O\left(\lambda^{-2 \mu-1}\right) \text { pour } \mu \neq \frac{1}{4} .
\end{aligned}
$$


REMARQUE. - En procédant de manière analogue dans le cas où $\nu$ est pair, on obtient les résultats suivants:

si $\mu=\frac{1}{4}, \quad|z|>M$,

$$
\begin{aligned}
& u_{\tau \pm 1, \alpha}(x, \lambda)=v_{\tau \pm 1}(x, \lambda)+e^{-\sigma z(x) / 2} \approx(x)^{\sigma k+\mu-\frac{1}{2}} 0(\lambda-2 \lg \lambda), \\
& u_{\tau \pm 1, \alpha}^{\prime}(x, \lambda)=v_{\tau \pm 1}^{\prime}(x, \lambda)+e^{-\sigma z(x) / 2} z(x)^{\sigma k+\mu-\frac{1}{2}} 0\left(\lambda-^{-1} \lg \lambda\right), \\
& u_{\tau, 0}(x, \lambda)=v_{\tau}(x, \lambda)+e^{\sigma z / 2} z^{-\sigma k+\mu-\frac{1}{2}} 0\left(\lambda-^{-2} \lg \lambda\right), \\
& u_{\tau, 0}^{\prime}(x, \lambda)=v_{\tau}^{\prime}(x, \lambda)+e^{\sigma z / 2} z^{-\sigma k+\mu-1}{ }_{2}^{1} 0\left(\lambda^{-1} \lg \lambda\right),
\end{aligned}
$$

si $\mu=\frac{1}{4}, \quad|z| \leq M$

$$
\begin{aligned}
& u_{\tau \pm 1}, \alpha \\
& u_{\tau \pm 1}^{\prime}(x, \lambda)=v_{\tau \pm 1}(x, \lambda)=v_{\tau \pm 1}^{\prime}(x, \lambda)+0\left(\lambda^{-3 / 2} \lg \lambda\right) \\
& u_{\tau, 0}(x, \lambda)=v_{\tau}(x, \lambda)+0\left(\lambda^{-2}\right) \\
& u_{\tau, 0}^{\prime}(x, \lambda)=v_{\tau}^{\prime}(x, \lambda)+0\left(\lambda^{-3 / 2}\right)
\end{aligned}
$$

si $\mu \neq \frac{1}{4},|z|>M$

$$
\begin{aligned}
& u_{\tau \pm 1, \alpha}(x, \lambda)=v_{\tau \pm 1}(x, \lambda)+e^{-\sigma z / 2} z^{\sigma k+\mu-\frac{1}{2}} 0\left(\lambda-^{2}\right), \\
& u_{\tau \pm 1, \alpha}^{\prime}(x, \lambda)=v_{\tau \pm 1}^{\prime}(x, \lambda)+e^{-\sigma z / 2} z^{\sigma k+\mu-\frac{1}{2}} 0(\lambda-1) \\
& u_{\tau, 0}(x, \lambda)=v_{\tau}(x, \lambda)+e^{\sigma z / 2} z^{-\sigma k+\mu-\frac{1}{2}} 0(\lambda-4 \mu-1) \\
& u_{\tau, 0}^{\prime}(x, \lambda)=v_{\tau}^{\prime}(x, \lambda)+e^{\sigma z / 2} z^{-\sigma k+\mu-\frac{1}{2}} 0(\lambda-4 \mu),
\end{aligned}
$$

si $\mu \neq \frac{1}{4}, \quad|z| \leq M$

$$
\begin{aligned}
& u_{\tau \pm 1, \alpha}(x, \lambda)=v_{\tau \pm 1}(x, \lambda)+0(\lambda-4 \mu-1), \\
& u_{\tau \pm 1, \alpha}^{\prime}(x, \lambda)=v_{\tau \pm 1}^{\prime}(x, \lambda)+0(\lambda-2 \mu-1), \\
& u_{\tau, 0}(x, \lambda)=v_{\tau}(x, \lambda)+0(\lambda-4 \mu-1), \\
& u_{\tau, 0}^{\prime}(x, \lambda)=v_{\tau}^{\prime}(x, \lambda)+0(\lambda-2 \mu-1) .
\end{aligned}
$$


Puisque

$$
\begin{gathered}
\mathcal{W}\left[u_{\tau \pm 1, \alpha}, u_{\tau, 0}\right]=\mathcal{W}\left[v_{\tau \pm 1}, v_{\tau}\right]+0(\lambda-3 / 2 \lg \lambda), \text { si } \mu=\frac{1}{4}, \\
\mathcal{W}\left[u_{\tau \pm 1, \alpha}, u_{\tau, 0}\right]=\mathcal{W}\left[v_{\tau \pm 1}, v_{\tau}\right]+O(\lambda-2 \mu-1 \lg \lambda), \text { si } \mu \neq \frac{1}{4},
\end{gathered}
$$

on voit que les solutions $u_{\tau \pm 1, \alpha}(x, \lambda)$ et $u_{\tau, 0}(x, \lambda)$ sont linéairement indépendantes.

VI. - Comportement asymptotique de deux solutions linéairement indépendantes de (1), pour $x \in[\alpha, \beta]$.

20. - Lorsque aucun des nombres $k \pm \mu+\frac{1}{2}$ n'est entier, on pent obtenir le comportement de deux solutions linéairement indépendantes de l'équation (1) dans tout l'intervalle $[\alpha, \beta]$, pour $|\lambda| \geq \Lambda$, arg $\lambda \in S_{p}(\lambda)$. Les deux solations en question sont les fonctions $u_{\rho, \beta}(x, \lambda)$ et $u_{\tau, \alpha}(x, \lambda)$ [resp. $u_{\rho}, \beta(x, \lambda), u_{\tau \pm 1, \alpha}(x, \lambda)$ ] lorsque $\vee$ est un entier impair [resp. $\vee$ pair].

Nous traiterons encore de façon détaillée le cas où $\vee$ est impair.

Le comportement de $u_{\rho, \beta}(x, \lambda)$ est donné par les relations $(67),(68)$ pour $|z|>M, x>0$ et celui de $u_{\pi, \alpha}(x, \lambda)$ par les relations (85), (86) pour $|z|>M$, $x<0$. Le wronskien de ces denx fonctions est indépendant de $x$ et pent être calculé en $x=0$, à l'aide des relations $(71),(72),(73),(74),(89),(90)$, (91) et (92). Il vient

$$
\mathcal{W}\left[u_{\beta, \beta} ; u_{\tau, \alpha}\right]=\mathcal{W}\left[v_{\rho}, v_{\tau}\right]+0(\lambda-s / 2 \cdot \lg \lambda)
$$

si $\mu=\frac{1}{4}, \nu$ impair,

$$
\mathcal{W}\left[u_{p, \beta} ; u_{\tau, \alpha}\right]=\mathcal{W}\left[v_{p}, v_{\tau}\right]+0(\lambda-2 \mu)
$$

si $\mu \neq \frac{1}{4}, \nu$ impair.

Les fonotions $u_{\rho, \beta}(x, \lambda)$ et $u_{\tau, \alpha}(x, \lambda)$ sont done linéairement indépendantes [d'après $(37),(39)]^{3}$ et $u_{\tau, \alpha}(x, \lambda)$ peut s'exprimer comme combinaison linéaire des fonctions indépendantes $u_{\rho, \beta}(x, \lambda)$ et $u_{\rho \pm 1, o}(x, \lambda)$; on a

$$
u_{\tau, a}(x, \lambda)=C_{11} u_{\rho, \beta}(x, \lambda)+C_{21} u_{p \pm 1, o}(x, \lambda)
$$

avec

$$
C_{11}=\frac{\mathcal{W}\left[u_{\tau, a} ; u_{\rho \pm 1,0}\right]}{\mathcal{W}\left[u_{\rho, \beta} ; u_{\rho \pm 1, \beta}\right]}, C_{21}=\frac{\mathcal{W}\left[u_{\rho, \beta} ; u_{\tau, \alpha}\right]}{\mathcal{W}\left[u_{\rho, \beta} ; u_{\rho \pm 1,0}\right]}
$$


Puisque

$$
\mathcal{W}\left[u_{\tau, \alpha} ; u_{\rho \pm 1,0}\right]=\mathcal{W}\left[v_{\tau}, v_{\rho \pm 1}\right]+0\left(\lambda^{-3 / 2} \cdot \lg \lambda\right)
$$

pour $\mu=\frac{1}{4}$

$$
\mathscr{W}\left[u_{\tau, \alpha} ; u_{p \pm 1,0}\right]=\mathscr{Q}\left[v_{\tau}, v_{p \pm 1}\right]+0\left(\lambda^{-2 \mu-1}\right),
$$

pour $\mu \neq \frac{1}{4}$

on déduit aisément des relations (99), (100), (82), (83) que

$$
\begin{aligned}
& C_{11}=\frac{\mathcal{W}\left[v_{\tau}, v_{\rho \pm 1}\right]}{\mathcal{W}\left[v_{\rho}, v_{\rho \pm 1}\right]}+0\left(\lambda^{-2} \lg \lambda\right) \text { si } \mu=\frac{1}{4}, \\
& C_{11}=\frac{\mathcal{W}\left[v_{\tau}, v_{\rho \pm 1}\right]}{\mathcal{W}\left[v_{\rho}, v_{\rho \pm 1}\right]}+O(\lambda-4 \mu-1) \text { si } \mu \neq \frac{1}{4}, \\
& C_{21}=\frac{\mathcal{W}\left[v_{\rho}, v_{\tau}\right]}{\mathcal{W}\left[v_{\rho}, v_{\rho \pm 1}\right]}+O\left(\lambda^{-2} \lg \lambda\right) \text { si } \mu=\frac{1}{4}, \\
& C_{21}=\frac{\mathcal{W}\left[v_{\rho}, v_{\tau}\right]}{\mathcal{W}\left[v_{\rho}, v_{\rho \pm 1}\right]}+0\left(\lambda^{-4 \mu-1)} \text { si } \mu \neq \frac{1}{4} .\right.
\end{aligned}
$$

En remplaçant dans (101), $C_{11}, C_{21}, u_{p, \beta}(x, \lambda)$ et $u_{p \pm 1, o}(x, \lambda)$ par leurs valeurs fournies respectivement par les relations (103) [ou (104)], (105) [ou (106)], (68) [ou (67)] et (78) [ou (79)] et en tenant compte de (37) et (39), on trouve

$$
\boldsymbol{u}_{\tau, \alpha}(x, \lambda)=v_{\tau}(x, \lambda)+v_{\rho}(x, \lambda) 0\left(\lambda-^{-2} \lg \lambda\right)+v_{\rho \pm 1}(x, \lambda) 0\left(\lambda-^{-2} \lg \lambda\right)
$$

si $\mu=\frac{1}{4}$,

$$
u_{\tau, a}(x, \lambda)=v_{\tau}(x, \lambda)+v_{\rho}(x, \lambda) 0\left(\lambda^{-4 \mu-1}\right)+v_{\rho \pm 1}(x, \lambda) 0\left(\lambda^{-4 p-2}\right), \quad \text { si } \quad \mu \neq \frac{1}{4},
$$

ou

$$
\left.u_{\tau, \alpha}(x, \lambda)=v_{\tau}(x, \lambda)+e_{2}^{\frac{1}{2} \sigma z} z^{-\sigma k+\mu+\frac{1}{2} 0(\lambda-2} \lg \lambda\right)
$$

si $\mu=\frac{1}{4}, x>0, \quad|z|>M$

$$
u_{\tau, \alpha}(x, \lambda)=v_{\tau}(x, \lambda)+e^{\frac{1}{2} \sigma z} z^{-\sigma k+p+\frac{1}{2}} 0(\lambda-4 \mu-1),
$$

si $\mu \neq \frac{1}{4}, x>0,|z|>M$, dans le secteur $S_{p}(\lambda)$. 
Rappelons que la solution $u_{\rho, \beta}(x, \lambda)$ linéairement indépendante de $u_{\tau}, \alpha(x, \lambda)$ est fournie par les relations (67) et (68).

21. - Lorsque $x$ est nógatif, les xôles de $\rho$ et $\tau$ sont inversés. En $x=0$, on obtient

$$
\begin{aligned}
& \mathcal{W}\left[u_{\tau, \alpha}, u_{\rho, \beta}\right]=\mathcal{W}\left[v_{\tau}, v_{\rho}\right]+0\left(\lambda^{-3 / 2} \lg \lambda\right) \text { si } \mu=\frac{1}{4}, \\
& \mathcal{W}\left[u_{\tau, \alpha}, u_{\rho, \beta}\right]=\mathcal{W}\left[v_{\tau}, v_{\rho}\right]+O\left(\lambda^{-2 \mu-1}\right) \text { si } \mu \neq \frac{1}{4}
\end{aligned}
$$

De plus, $u_{\rho}, \beta(x, \lambda)$ peut s'exprimer comme combinaison linéaire des fonctions indépendantes $u_{\tau, \alpha}(x, \lambda), u_{\tau \pm 1}, o(x, \lambda)$; on a

$$
u_{p, \beta}(x, \lambda)=C_{12} u_{\tau, \alpha}(x, \lambda)+C_{22} u_{\tau \pm 1,0}(x, \lambda)
$$

avec

$$
C_{12}=\frac{\mathcal{W}\left[\boldsymbol{u}_{p, \beta} ; \boldsymbol{u}_{\tau \pm 1,0}\right]}{\mathcal{W}\left[\boldsymbol{u}_{\tau, \alpha} ; \boldsymbol{u}_{\tau \pm 1,0}\right]} ; \quad C_{22}=\frac{\mathcal{W}\left[u_{\tau, \alpha} ; u_{\rho, \beta}\right]}{\mathcal{W}\left[u_{\tau, \alpha} ; u_{\tau \pm 1,0}\right]}
$$

En procédant comme au paragraphe 19, on obtient finalement

$$
u_{\rho, \beta}(x, \lambda)=v_{\rho}(x, \lambda)+e^{\frac{1}{2} \sigma z} z^{-\sigma k+\mu-\frac{1}{2}} O(\lambda-2 \lg \lambda),
$$

si $\mu=\frac{1}{4}, x<0, \quad|z|>M$

$$
u_{\rho, \beta}(x, \lambda)=v_{\rho}(x, \lambda)+e^{\frac{1}{2} \sigma z} z^{-\sigma k+\beta-\frac{1}{2}} 0(\lambda-4 \mu),
$$

si $\mu \neq \frac{1}{4}, x<0, \quad|z|>M$ dans le secteur $S_{\rho}(\lambda)$.

Dans ce cas, la solution $\boldsymbol{u}_{\tau, \alpha}(x, \lambda)$ linéairement indépendante de $\boldsymbol{u}_{\beta}, \beta(x, \lambda)$ est fournie par les relations (85), (86).

Pour $|z| \leq M$, on a enfin dans $S_{p}(\lambda)$

$$
\begin{aligned}
& u_{\rho, \beta}(x, \lambda)=v_{\rho}(x, \lambda)+0\left(\lambda^{-2} \lg \lambda\right), \text { pour } \mu=\frac{1}{4}, \\
& u_{\rho, \beta}(x, \lambda)=v_{\rho}(x, \lambda)+0\left(\lambda^{-4 \mu-1}\right), \\
& u_{\tau, \alpha}(x, \lambda)=v_{\tau}(x, \lambda)+0\left(\lambda^{-2} \lg \lambda\right), \text { pour } \mu \neq \frac{1}{4}, \\
& u_{\tau, \alpha}(x, \lambda)=v_{\tau}(x, \lambda)+0(\lambda-4 \mu-1), \text { pour } \mu \neq \frac{1}{4},
\end{aligned}
$$


22. - Dans le cas où $v$ est pair, on obtient pour $x>0, \arg \lambda \in S_{p}(\lambda)$,

si $\mu=\frac{1}{4}, \quad|z|>M$,

$u_{\tau \pm 1, \alpha}(x, \lambda)=v_{\tau \pm 1}(x, \lambda)+e^{\frac{1}{2} \sigma z} z-\sigma k+\mu-\frac{1}{2} 0(\lambda-2 \lg \lambda)$,
si $\mu \neq \frac{1}{4}, \quad|z|>M$,

$$
\left.u_{\tau \pm 1, \alpha}(x, \lambda)=v_{\tau \pm 1}(x, \lambda)+e^{\frac{1}{2} \sigma z z^{-\sigma k+\mu-\frac{1}{2}} 0(\lambda-4 \mu-1}\right)
$$

La solution $u_{\rho, \beta}(x, \lambda)$ linéairement indépendante de $u_{\tau \pm 1, \alpha}(x, \lambda)$ est fournie par les relations $(67)$ et $(68)$.

Pour $x<0$, il vient

si $\mu=\frac{1}{4},|z|>M$,

$$
u_{\rho, \beta}(x, \lambda)=v_{\rho}(x, \lambda)+e^{\frac{1}{2} \sigma z} z^{-\sigma k+\mu-\frac{1}{2}} 0(\lambda-2 \lg \lambda),
$$

si $\mu \neq \frac{1}{4}, \quad|z|>M$,

$$
u_{\rho, \beta}(x, \lambda)=v_{\rho}(x, \lambda)+e_{2}^{\frac{1}{2} \sigma z} z^{-\sigma k+\mu-\frac{1}{2}} 0\left(\lambda^{-4 \mu-1}\right) .
$$

La solution $u_{\tau \pm 1, \alpha}(x, \lambda)$ linéairement indépendante de $u_{\rho, \beta}(x, \lambda)$ est donnée par les relations

$$
\begin{aligned}
& \left.\quad u_{\tau \pm 1, \alpha}(x, \lambda)=v_{\tau \pm 1}(x, \lambda)+e^{-\frac{1}{2} \sigma z \sigma k+\mu-\frac{1}{2} O(\lambda-2} \lg \lambda\right), \\
& \text { si } \mu=\frac{1}{4}, \quad|z|>M,
\end{aligned}
$$

$$
u_{\tau \pm 1, \alpha}(x, \lambda)=v_{\tau \pm 1}(x, \lambda)+e^{-\frac{1}{2} \sigma z z^{\sigma k+\mu-\frac{1}{2}} 0(\lambda-2),}
$$

si $\mu \neq \frac{1}{4},|z|>M$.

Pour $|z| \leq M$, on a done dans $S_{p}(\lambda)$

$$
\begin{aligned}
& u_{\rho, \beta}(x, \lambda)=v_{\rho}(x, \lambda)+0\left(\lambda^{-2} \lg \lambda\right), \quad \text { si } \mu=\frac{1}{4}, \\
& u_{\rho, \beta}(z, \lambda)=v_{\rho}(x, \lambda)+0\left(\lambda^{-4 \mu-1}\right), \quad \text { si } \mu \neq \frac{1}{4}, \\
& u_{\tau \pm 1, \alpha}(x, \lambda)=v_{\tau \pm 1}(x, \lambda)+0\left(\lambda^{-2} \lg \lambda\right), \text { si } \mu=\frac{1}{4}, \\
& u_{\tau \pm 1, \alpha}(x, \lambda)=v_{\tau \pm 1}(x, \lambda)+0(\lambda-4 \beta-1), \quad \text { si } \mu \neq \frac{1}{4} .
\end{aligned}
$$


200 J. LonbET-GoFfar: Etude des solutions d'équations différentielles, etc.

\section{BIBLIOGRAPHIE}

[1] H. Buchноцz, Die Konfhente hypergeometrische Funktion, Springer Verlag 1953.

[2] A. ERdḱlyr, Asymptotic expansions, "Dover Publications», 1956.

[3] R. LANGER, a) On the asymptotic solutions of ordinary differential equations with an application to the Bessel Functions of large order, "Transactions of the American Mathematical Society $\mathrm{n}^{\circ} 33,(1931)$.

b) The asymptotic solutions of certain linear ordinary differential equations of the second order, "Transactions of the American Mathematical Society $n^{\circ} 36$ (1934).

[4] R. Mc KeLvey, a) The solutions of second order linear ordinary differential equations about a turning point of order two, «Transactions of the American Mathematical Society $\mathrm{n}^{\circ} 79$, (1955).

b) Solution about a singular point of a linear differential equation involving a large parameter, "Transactions of the American Mathematical Society $n^{\circ} 92$ * (1959).

[5] L. J. SLATER, Confluent hypergeometric Functions, "Cambridge University Press". 